\title{
Protée
}

\section{Analyse des images scientifiques par le concept d'observation}

\section{Vincent Israël-Jost}

Volume 37, numéro 3, hiver 2009

Regards croisés sur les images scientifiques

URI : https://id.erudit.org/iderudit/038801ar

DOI : https://doi.org/10.7202/038801ar

Aller au sommaire du numéro

Éditeur(s)

Département des arts et lettres - Université du Québec à Chicoutimi

\section{ISSN}

0300-3523 (imprimé)

1708-2307 (numérique)

Découvrir la revue

\section{Citer cet article}

Israël-Jost, V. (2009). Analyse des images scientifiques par le concept d’observation. Protée, 37(3), 9-17. https://doi.org/10.7202/038801ar

\section{Résumé de l'article}

Une partie importante des images scientifiques sont produites dans le but d'observer des entités ou des phénomènes, c'est-à-dire d'obtenir à leur sujet une connaissance sûre, acquise par des moyens aussi directs que possible. $\mathrm{Ce}$ rôle des images et des instruments qui les produisent a été évalué par les philosophes, en particulier depuis le début des années 1980, pour tenter de comprendre si l'emploi du terme « observer » est justifié dans le cas où une chaîne complexe d'instrumentation est mise en place pour produire des images. Dans ce cas, en effet, on peut craindre une contamination des images à la fois par des artefacts, en cas de défaut du dispositif expérimental, et par les théories sur lesquelles repose ce même dispositif. Sans se prononcer sur la validité des instruments pour l'observation dans le présent travail, nous décrivons en quoi les analyses philosophiques relatives à cette question sont insuffisantes pour rendre compte de l'utilisation réelle qui est faite des images par les scientifiques. Nous tirons deux éléments importants de cette analyse : d'une part, la nécessité de considérer une image à la lumière d'un contexte scientifique spécifique qui donne l'objectif de la démarche expérimentale et précise ce que l'on tente d'observer et, d'autre part, le fait que des phénomènes de la plus grande variété peuvent être représentés sur une image. Ce deuxième aspect s'oppose à l'idée très généralement partagée par les philosophes que seules les propriétés spatiales des entités pourraient être explorées par l'image. Pour importants que soient ces aspects de forme, d'échelle et de localisation, il faut les compléter d'abord par la possibilité d'explorer des propriétés physiques détectables, telles que la luminosité, la radioactivité, etc., et ensuite, en introduisant la notion de propriétés de haut niveau, propres à chaque domaine des sciences. Nous ouvrons ainsi la question de savoir si l'on peut observer des phénomènes aussi divers que la perfusion cardiaque, la production d'énergie dans le noyau solaire, la région des émotions sur des images cérébrales de résonance magnétique fonctionnelle, etc.
Ce document est protégé par la loi sur le droit d'auteur. L'utilisation des services d’Érudit (y compris la reproduction) est assujettie à sa politique d'utilisation que vous pouvez consulter en ligne.

https://apropos.erudit.org/fr/usagers/politique-dutilisation/ 


\section{ANALYSE DES IMAGES SCIENTIFIQUES PAR LE CONCEPT D'OBSERVATION}

VINCENT ISRAËL-JOST

Une partie importante des images produites par les scientifiques le sont aujourd'hui à l'aide d'instruments tels que microscope, télescope, scanner et autres appareils d'imagerie. Ceux-ci s'appuient sur des sources de rayonnement électromagnétique (en imagerie par ondes radio, par rayons X, etc.) ou mécaniques (en échographie) et renseignent le plus souvent sur des phénomènes imperceptibles par les sens non assistés. Le point commun de ces images est qu'elles sont généralement utilisées comme preuve de l'existence ou de l'intensité d'un phénomène. Ce rôle de preuve est traditionnellement attaché au concept d'observation qui est essentiel dans les sciences empiriques.

Il est courant de caractériser l'observation, dans sa relation à la théorie, par le rôle qu'elle joue à la fois en amont et en aval de celle-ci. En amont, l'observation est la collecte de données sur lesquelles des hypothèses descriptives, classificatoires et, dans une certaine mesure, explicatives peuvent être construites. Il s'agit là de ce que les empiristes logiques ont décrit comme étant le contexte de découverte. En aval, l'observation sert à la mise à l'épreuve des prédictions faites à partir de ces hypothèses, dans un contexte de justification. On peut donc voir l'observation comme une activité qui encadre littéralement la production d'hypothèses théoriques par les scientifiques. Bien que cette vue apparaisse couramment chez les philosophes des sciences, elle rend le concept d'observation inapplicable dans un cadre non théorique tel que celui de la médecine clinique de routine, dans lequel le médecin ne cherche pas à observer tel ou tel organe chez son patient dans le but de construire ou de justifier une théorie. C'est la raison pour laquelle, pour utiliser le concept d'observation dans l'analyse des images scientifiques dans leur plus grande généralité, nous devons utiliser une définition plus large de l'observation qui s'accorde ou non avec des contextes théoriques. Ainsi, le propre de l'observation est, dans tous les cas, d'apporter une connaissance fiable et objective relative au monde extérieur, ce qu'exprime Claude Bernard lorsqu'il définit l'observation comme "la constatation exacte d'un fait à l'aide de moyens d'investigation et d'études appropriées à cette constatation" ([1865] 1966: 40).

L'une des qualités attendues de la collecte des données pour l'observation est qu'elle limite la présence d'éléments susceptibles de dénaturer les faits, ce pourquoi l'on essaie de la réaliser de manière aussi directe que possible. Il n'est donc pas surprenant que la perception humaine ait traditionnellement reçu le statut de moyen 
privilégié pour l'observation, dans la mesure où tout recours à l'instrumentation induit une étape supplémentaire dans le recueil des données, ces dernières étant alors davantage susceptibles d'être contaminées par des artefacts. Pourtant, face au nombre accru d'images produites avec des instruments qui mettent en jeu un grand nombre d'étapes dans la production des données, mais que les scientifiques utilisent pourtant comme la preuve irréfutable de faits de nature variée - souvent d'ailleurs en utilisant le terme «observation»-, un débat philosophique concernant le statut des données produites par des instruments s'est ouvert, prenant une ampleur particulière au début des années 1980. Ce débat met aux prises les partisans de la position traditionnelle, dans laquelle la perception humaine conserve un statut privilégié, et ceux qui, en redéfinissant le concept d'observation, tentent de donner aux images produites par des instruments un statut équivalent à celui de l'expérience directe, par les sens non assistés. Les deux camps s'accordent cependant à considérer la photographie et les instruments optiques qui ne permettent que d'enregistrer un phénomène déjà visible comme des moyens valides pour l'observation ${ }^{1}$ En les utilisant, les scientifiques s'assurent en effet de pouvoir comparer les images obtenues avec leur expérience directe et la fiabilité de ces instruments peut donc aisément être établie. En revanche, les techniques d'imagerie qui produisent des données relatives à des phénomènes non observables par la perception seule ne tolèrent pas cette mise à l'épreuve comparative, et leur fiabilité, si elle doit être démontrée, demande une méthode différente.

L'ambition du présent travail est de discuter de manière préliminaire le contenu d'une méthode pour s'assurer de la fiabilité des images produites par les instruments. Ainsi, notre objectif est non pas de répondre ici à la question de la validité des instruments comme moyens pour l'observation mais, en nous penchant sur cette question, d'établir un argument essentiel à la construction d'une réponse. Notre point de départ est le constat que les analyses trouvées chez les philosophes qui se sont exprimés sur cette question se polarisent, quelle que soit leur position, sur les seules propriétés géométriques des images. C'est ainsi la concordance entre la structure d'une entité (l'objet d'étude) et les formes perçues sur l'image (les «traits» de l'image, les contours qui s'y dessinent) qui, dans la plupart de ces travaux, est l'axe central de la question de la fiabilité. Les notions de forme, de structure, de géométrie, qui dans notre propos peuvent être considérées comme à peu près synonymes, y prennent un poids considérable, traduisant, à notre sens, un appauvrissement relatif à la variété des phénomènes que les scientifiques affirment observer. En mettant l'accent sur les seules propriétés spatiales des entités étudiées avec des instruments, nous verrons que c'est la fiabilité de la détection et de la transmission d'une information qui est essentiellement traitée, c'est-à-dire la question des moyens de l'observation ou comment l'on observe. En revanche, la question du contenu de l'observation ou ce que l'on observe est quelque peu délaissée.

Pour établir l'insuffisance de ces analyses, nous identifions, en premier lieu, des facteurs pouvant expliquer leur restriction aux seules propriétés spatiales des entités étudiées par les scientifiques. Nous discutons ensuite les positions du représentant principal de chaque camp du débat mentionné: Bas C. van Fraassen chez les partisans (peu nombreux) d'un concept d'observation fondé exclusivement sur la perception non assistée et Ian Hacking qui, dans sa discussion relative au microscope, défend une certaine forme de réalisme vis-à-vis des entités vues au travers de l'instrument. Pour antagonistes qu'elles soient, nous démontrons que ces positions s'appuient sur une vue similaire de l'observation, limitée aux seules propriétés structurales des entités. Nous concluons sur la nécessité de discuter des propriétés autres que structurales pour trancher le débat sur l'utilisation des instruments pour l'observation, en tenant compte de la grande variété des phénomènes qui font l'objet d'études scientifiques.

\section{L'INTERPRÉTATION GÉOMÉTRIQUE DES IMAGES PRODUITES PAR LES INSTRUMENTS}

La tentation de fonder l'interprétation des images scientifiques produites par les instruments sur leurs seules propriétés géométriques trouve principalement sa source dans l'idée couramment répandue 
qu'interpréter une image scientifique reviendrait à identifier les entités qui y sont représentées. Quiconque adopte cette position se met alors en devoir d'associer aux formes perçues sur l'image celles connues ou supposées d'entités pour réaliser cette identification. Ainsi, face aux images produites par les techniques d'imagerie contemporaines, le premier réflexe du lecteur non initié consiste à parvenir à identifier une entité à travers la reconnaissance d'une forme familière. Sur une image par résonance magnétique (IRM) par exemple, il n'est guère besoin de posséder des connaissances très avancées en anatomie pour déterminer, à la vue des circonvolutions caractéristiques, qu'il s'agit d'une image du cerveau. Il en est de même pour des images astrophysiques dans lesquelles nous interprétons la présence de spirales lumineuses comme des galaxies ou pour des images réalisées au microscope qui montreraient des structures circulaires ayant une frontière très précise: il s'agit sans doute de cellules. Le spécialiste de ces techniques est supposé jouir d'une expertise bien supérieure. Il sait, d'une part, distinguer avec certitude des entités que le profane pourrait confondre (le microbe et la bactérie par exemple) et connaît, par ailleurs, les détails du processus par lequel ces images sont acquises, ce qui lui donne la possibilité de comprendre, notamment, que certains traits ou certaines déformations de l'image sont produits par l'instrument. Enfin, à sa capacité de reconnaître une entité s'ajoute celle d'en distinguer des sous-parties telles que, dans le cas d'images cérébrales, la matière blanche, la matière grise, les ventricules, etc. Il n'en demeure pas moins que les propriétés spatiales de l'image sont effectivement suffisantes dans cette vue pour justifier du rôle des techniques d'imagerie dans l'observation, un rôle dévolu essentiellement aux questions d'existence, de reconnaissance et de localisation d'entités.

L'une des causes possibles de la popularité de cette idée, que nous pouvons relever dans le contexte précis de l'utilisation d'instruments pour l'observation, est l'analogie fréquemment rencontrée entre l'expérience visuelle et celle que l'on a en regardant les images produites par un instrument (ou en regardant directement à travers un instrument lorsque c'est possible), aussi bien dans les discours des scientifiques que chez les philosophes. Chez ces derniers, une préoccupation majeure est précisément de décider si ces techniques sont aussi fiables que la vision - se demandant, par exemple, si l'on peut «voir» à travers un microscope ${ }^{2}$. Or, la volonté de conserver le vocabulaire propre à la vision présuppose que, s'il faut chercher une différence entre notre expérience perceptive et l'expérience que nous avons en regardant les images produites par des instruments, cette différence ne saurait se situer sur le plan phénoménologique. Autrement dit, nous voyons des phénomènes mettant en jeu des propriétés identiques dans les deux cas, et notre attention porte seulement sur des entités différentes. Les techniques d'imagerie seraient donc destinées à nous apporter une connaissance relative aux entités qui sont autrement inobservables, c'est-à-dire les entités microscopiques, ou très lointaines ou encore celles qui sont enfouies dans de la matière opaque, mais cette connaissance concernerait toujours des propriétés qui sont accessibles à la vue, celles auxquelles nous accéderions si nous pouvions voir ces entités sans instrument.

À travers l'interprétation géométrique des images se dessine ainsi une position qui reconnaît en l'entité l'objet canonique de l'observation. Cette notion d'entité ne possède cependant qu'un certain nombre de propriétés primaires, qui sont toutes essentiellement spatiales: la forme, la position et la taille ${ }^{3}$. La forme est la propriété essentielle à la reconnaissance d'une entité et permet donc de répondre aux questions d'existence ou de présence dans le champ de vue de l'instrument. La position permet de suivre une entité dans l'espace et joue un rôle important dans l'observation des phénomènes dynamiques puisque connaître la position à des instants différents, c'est connaître le mouvement. Enfin la taille est, parmi ces propriétés fondamentales, celle qui explique le mieux le recours à différents instruments adaptés aux dimensions microscopiques ou macroscopiques des entités. Si ces propriétés sont, comme le conçoivent explicitement ou implicitement nombre de philosophes, les seules dont l'observation 
doive rendre compte dans le contexte scientifique, alors le problème de l'utilisation des instruments se résume à la possibilité de connaître, de manière sûre, la relation géométrique qui lie les points du champ de vue de l'instrument à ceux de l'image qu'il produit.

\section{LES INSTRUMENTS DANS L'OBSERVATION CHEZ LES PHILOSOPHES}

L'exposé des différents travaux philosophiques ayant soutenu ou rejeté l'utilisation d'instruments pour l'observation serait bien trop long pour trouver sa place ici. Pour la question qui nous intéresse, qui consiste à déterminer si une discussion, limitée aux seules propriétés géométriques des images et des entités auxquelles ces images se rapportent, est suffisante pour rendre compte de l'activité d'observer dans les sciences, il nous suffira d'examiner les positions adoptées par les représentants principaux de chaque camp, généralement reprises sur ce point précis par leurs partisans.

Chez van Fraassen, l'observabilité est définie uniquement pour des entités. Pour lui, une entité est «observable» si elle peut être directement observée par la perception non assistée. Une entité peut bien être hors de portée, elle demeure observable dès lors qu'il existe des conditions dans lesquelles elle est accessible à notre perception. Il donne ainsi l'exemple des lunes de Jupiter qui, en dépit de leur distance par rapport à la terre, pourraient être observées à l'œil nu par des astronautes à bord d'un vaisseau spatial et sont, par conséquent, observables dans sa conception du terme. En sus, il accepte les images fournies par un télescope pour l'observation, même si la comparaison avec l'expérience directe n'est pas réalisée, du moment qu'elle est réalisable en principe (Fraassen, 1980:16). En revanche, nous n'avons pas la possibilité de nous rapetisser pour observer des entités microscopiques qui sont donc définitivement reléguées dans la catégorie «inobservable».

Nous reconnaissons dans cette position deux aspects évoqués plus haut: la comparaison avec la vision à l'œil nu comme critère essentiel visant à établir le statut d'observation des images fournies par un instrument et son corollaire, l'entité munie de ses seules qualités géométriques, posée comme étant l'objet canonique de l'observation. Ses failles apparaissent donc rapidement dès lors que l'on tente de l'appliquer à des entités pour lesquelles les scientifiques étudient des propriétés non géométriques. C'est le cas en médecine, où l'étude de l'anatomie humaine couvre le savoir lié à la forme, la structure et la localisation des différentes parties du corps, et se distingue en cela de la physiologie qui en étudie les processus normaux. Suivant donc la position de van Fraassen, nous dirions que le cœur, par exemple, est observable parce que nous pouvons non seulement imaginer le voir en principe (en incisant la poitrine d'un patient), mais il est parfois vu réellement par des chirurgiens qui procèdent à une opération. L'ayant classé dans la catégorie "observable», nous poursuivons en affirmant que les différents moyens qui s'offrent à nous pour faire des images du cour, tels que l'échographie, le scanner ou la médecine nucléaire, sont valides ou peuvent être aisément validés pour l'observation de cet organe, de la même manière que van Fraassen accepte que l'on utilise un télescope pour observer les lunes de Jupiter. Pourtant, certaines de ces techniques sont destinées à renseigner sur des propriétés fonctionnelles du cœur, comme en médecine nucléaire où la tomographie d'émission monophotonique (TEMP) permet de réaliser une image de la perfusion myocardique. On peut raisonnablement penser que van Fraassen réfuterait cette possibilité d'observer la perfusion du cour par des moyens extrêmement indirects, puisque mettant en jeu l'injection d'un traceur radioactif émetteur de photons gamma, un type de rayonnement que nous ne pouvons pas voir et une lourde phase de calculs réalisés à partir des données pour reconstruire une vue tridimensionnelle du cœur; mais il s'agit pourtant d'une conclusion obtenue à partir de sa propre construction de l'observabilité et de ce qui compte comme un cas d'observation. L'ambiguité de sa position est, on le voit, générée essentiellement par le fait que l'entité (dans sa conception simplifiée, géométrique) y a pris le pas sur le phénomène dans le discours sur l'observation.

Opposé à l'anti-réalisme de van Fraassen concernant ce que montrent les images produites par des instruments, Ian Hacking s'est concentré sur 
le microscope qui, affirme-t-il, produit des données aussi fiables que celles qui sont collectées durant le processus de la vision, dès lors que l'instrument et l'œil sont utilisés dans des conditions de lumière et d'échelle appropriées. Le premier élément en faveur de cette thèse est le fait que les images sont produites par le microscope à travers une série d'événements physiques, conduisant dans la majorité des cas à une «bonne carte» de la réalité, c'est-à-dire à une image sur laquelle l'agencement spatial des différentes structures d'un objet est correctement reproduit (Hacking, [1981] 2004: 272).

Cependant, cette condition n'est pas suffisante pour garantir l'obtention d'une bonne carte dans tous les cas. Les instruments sont des sources d'artefacts et induisent des erreurs, des distorsions, des éléments ajoutés sur l'image qui doivent être distingués de ceux correspondant à l'objet étudié, ce pour quoi Hacking propose deux moyens de vérifier l'instrument.

Le premier est d'expérimenter, en passant sous microscope, un objet qui a été spécialement conçu pour cette vérification. L'exemple qu'il donne est celui d'une micro-grille, dont la structure est parfaitement connue à l'avance et peut être retrouvée au microscope (ibid. : 264). Puisque le microscope produit une image dans laquelle la structure de la micro-grille est correctement reproduite, on peut légitimement inférer que le même instrument possède la propriété de produire une bonne carte, indépendamment de l'objet étudié. Mais, de cet exemple, il ressort aussi que la discussion est limitée aux propriétés géométriques. Seule la conservation de la forme est vérifiée, tandis que les autres propriétés pouvant être associées aux entités ne sont pas mentionnées.

La deuxième possibilité que décrit Hacking repose sur l'utilisation de plusieurs microscopes fonctionnant sur des principes physiques différents, pour vérifier que, dans tous les cas, une image présentant la même structure est obtenue lorsque le même objet est étudié (la même micro-grille par exemple). Si effectivement la même structure est retrouvée chaque fois, il ne pourrait s'agir d'une coïncidence puisque les processus physiques différents qui sont associés à ces divers instruments ne pourraient conduire au même artefact qu'avec une chance infime (ibid.: 264-265). L'argument est encore une fois recevable, mais toujours limité aux seules propriétés géométriques. Pire, alors que Hacking reconnaît l'existence d'une grande variété d'instruments basés sur des fonctionnements distincts, et qui détectent des propriétés différentes de la matière, insiste-t-il lui-même, il réduit les capacités exploratoires de ces instruments à leur facteur commun: la représentation de la structure.

Cette limitation chez Hacking est certes consciente puisque, en ce qui concerne le microscope, il se prétend d'un réalisme scientifique concernant les structures des entités et rien de plus. Il est également vrai que la microscopie demeure une technique essentiellement morphologique, destinée à apporter une connaissance relative aux structures, en biologie cellulaire. Néanmoins, parmi les philosophes qui ont repris l'argumentaire de Hacking pour l'adapter à d'autres techniques, peu se sont résolus à introduire d'autres propriétés, et la discussion du réalisme scientifique portant au-delà des seules propriétés structurales n'a guère avancé depuis.

Paul Humphreys a reconnu cette même limitation et s'est demandé s'il n'y aurait pas des entités à la fois observables et inobservables $(2004: 23)^{4}$, c'està-dire observables sous certains aspects mais pas sous d'autres. Cela le conduit à renoncer à penser l'observation comme s'appliquant à des entités, pour lesquelles les questions se limiteraient à l'existence et à la localisation, préférant se référer aux propriétés:

Des différends existent à propos de la réalité d'objets ou de types d'objets: est-ce que les prions existent? Y a-t-il une planète au-delà de Neptune? Quelle preuve y avait-il à la fin du XIXe siècle en faveur de l'existence des atomes? Est-ce que les émotions existent séparément de leurs états mentaux associés? et ainsi de suite. Ces exemples peuvent être trompeurs, car les considérations sur le réalisme concernent également l'existence de propriétés. Comment savons-nous que le spin quantique existe? Y a-t-il une propriété telle que l'aliénation dans la société? Est-ce que la liaison covalente est une propriété purement chimique? [...] L'ensemble du débat sur les observables a été biaisé par une attention excessive portée sur la taille et les distances relatives comme mesures de substitution du degré de difficulté à observer des objets.

(Ibid.: 23 ; notre traduction $)^{5}$ 
Mais les préoccupations des scientifiques concernant les propriétés de natures diverses ne sont pas la seule raison pour laquelle il serait plus adéquat, selon Humphreys, d'en faire l'objet de l'observation. L'utilisation même d'instruments impose que l'on détecte des propriétés (chaleur, radioactivité, luminosité, etc.) et non des entités puisque nous n'avons pas de détecteurs de planètes, de cerveau ou de mitochondries. Il donne ainsi l'image d'une exploration progressive des entités, qui dépend du développement de nouveaux instruments:

Le processus de découverte concernant des objets consiste en des types spécialisés d'instruments détectant une ou plusieurs propriétés qui constituent ce que nous considérons être cet objet avec, par conséquent, la possibilité de détecter de plus en plus de propriétés composant cette entité à mesure que de nouvelles techniques apparaissent.

(Ibid.: 25 ; notre traduction) ${ }^{6}$

Dans cette vue, une image produite par un instrument représente la propriété physique qu'il détecte, telle qu'elle est distribuée sur son champ de vue. Nous appelons une telle représentation d'une unique propriété une carte de cette propriété. La multiplication des instruments s'explique par la possibilité qu'ils offrent de produire des cartes de propriétés physiques différentes, qui sont bien entendu spatialement corrélées. Cette corrélation est l'élément qui permettait à Ian Hacking de s'assurer que la structure géométrique d'une entité est bien retrouvée indépendamment par des instruments basés sur des principes différents. Cependant, la corrélation se distingue de l'identité et Humphreys tient compte de cela en invoquant la nature variée de l'information portée par des images qui n'ont pas été produites avec le même type d'instrument. Toutes ces cartes sont donc superposables, tout en pouvant se différencier de façon extrêmement marquée, comme pourraient l'être des cartes du monde présentant des informations politiques, géographiques, économiques, etc.

\section{DE LA VARIÉTÉ INSTRUMENTALE}

\section{À LA VARIÉTÉ PHÉNOMÉNALE}

Un bénéfice important de la position de Paul Humphreys est qu'elle permet d'expliquer la variété des techniques instrumentales qui sont développées par l'intérêt qu'elles présentent pour explorer des propriétés nouvelles. Aux propriétés spatiales des entités, il ajoute ainsi tout un faisceau de propriétés physiques mesurables auxquelles sont associées autant d'instruments. Pourtant, en dépit de ce progrès, cette position ne donne encore qu'une vue partielle de l'observation, puisque les scientifiques ne limitent pas leurs investigations aux seules propriétés physiques, mesurables par un détecteur. L'un des aspects frappants des techniques d'imagerie actuelles est précisément que, par leur intermédiaire, les scientifiques affirment avoir une connaissance relative à des propriétés qui sont propres à un domaine scientifique particulier, par exemple les propriétés biologiques, physiologiques, cognitives, etc.

Donc, si Humphreys a doublement raison, d'une part de souligner qu'il est plus adéquat, pour rendre compte des objectifs des scientifiques, de parler d'observation en termes de propriétés plutôt que d'entités, d'autre part de rappeler que nos instruments détectent des propriétés et non des entités, il faudrait néanmoins procéder à une distinction entre les propriétés détectées, qui sont toujours des propriétés physiques que nous pouvons qualifier de «bas niveau " 7 , et les propriétés (prétendument) observées. Ces dernières sont parfois identiques aux premières, lorsque les scientifiques sont directement intéressés par une propriété physique, comme la luminosité d'un astre, mais, dans beaucoup d'autres situations, une image présentant une carte de propriété physique est interprétée comme se référant à une propriété de plus haut niveau, propre à un domaine scientifique particulier.

Pour illustrer cette distinction entre propriétés de bas niveau et de haut niveau, nous pouvons revenir sur l'exemple de l'imagerie TEMP, qui repose sur l'administration d'un traceur radioactif ou radiotraceur, c'est-à-dire une molécule qui s'inscrit dans un métabolisme particulier de l'organisme, et sur laquelle est fixé un atome radioactif. Lors d'un examen cardiaque, après injection du radiotraceur par voie veineuse, celui-ci suit le flux sanguin, et la diminution ou l'absence de sa fixation sur la paroi myocardique sont révélatrices de problèmes 
coronariens qui correspondent respectivement à une ischémie (artère partiellement obstruée) ou à une nécrose (obstruction totale). L'image obtenue est formée à partir du rayonnement gamma émis par l'atome radioactif qui est fixé sur le radiotraceur et peut donc être interprétée comme une carte de la radioactivité présente dans l'organisme après injection du radiotraceur. Il s'agit là de la propriété physique (bas niveau) que l'on détecte, à l'aide d'une gammacaméra. Pourtant, bien que les médecins qui utilisent ces images pour leur diagnostic soient conscients que la lecture de l'image comme "carte de la radioactivité" est possible, ils préfèrent généralement parler d'une image de perfusion myocardique, car c'est là le phénomène qu'ils cherchent à observer. Il s'agit dans les deux cas de propriétés, mais d'ordre différent, physique dans un cas et physiologique dans l'autre. On note également que l'entité qui est explorée n'apparaît dans cette description de l'image que dans une forme adjectivale, qui indique à quel organe la propriété de perfusion se réfère. Cette forme indique le caractère secondaire de la notion d'entité dans ce type d'exploration dans lequel l'existence, la localisation et la forme sont des questions dont la réponse est déjà supposée connue.

Il ressort de cette analyse qu'il serait saugrenu de se prononcer sur le statut d'observation d'une image scientifique de manière absolue, c'est-à-dire indépendamment d'un contexte qui donne les objectifs de la démarche. Une image d'IRM cérébrale fonctionnelle pourrait ainsi permettre de s'assurer de la présence d'un cerveau dans une boîte crânienne, comptant à ce titre comme une "observation» du cerveau acceptée par l'ensemble de la communauté scientifique. En revanche, si cette même image est produite dans l'idée d'établir, par exemple, quelle est «la région des émotions" dans le cerveau, son statut d'observation devient beaucoup plus difficile à déterminer. Une construction explicative plus élaborée est alors nécessaire pour justifier du statut d'observation de ce que nous pouvons appeler les propriétés de haut niveau, par rapport aux propriétés de bas niveau, c'est-à-dire la propriété physique détectée et les propriétés spatiales. Il faudrait donc rendre compte de la manière dont ces propriétés de haut niveau peuvent être observées à partir des propriétés de bas niveau.

Il est clair que, dans ce projet, une explication relative aux propriétés de bas niveau est nécessaire en premier lieu et que celle-ci repose sur la fiabilité de l'instrument. Il serait par exemple difficile d'affirmer que l'on observe la «région des émotions» dans le cerveau avant de nous être assurés que l'instrument que nous utilisons délivre une bonne carte du cerveau, avec un agencement correct des différentes structures visibles. Cela montre en quoi les analyses de Hacking et de Humphreys étaient sur la bonne voie, en offrant au moins une condition nécessaire pour qu'une image puisse servir à l'observation. L'analyse doit cependant être poursuivie pour établir un lien entre les propriétés de bas niveau et celles de haut niveau. Dans la mesure où ces dernières sont spécifiques à des domaines de la science très divers, nous ne saurions rendre ici notre analyse générale, mais pouvons illustrer la forme qu'elle prend dans deux cas particuliers et en tirer quelques enseignements.

Dans le premier, nous poursuivons sur l'observation de la perfusion du myocarde (propriété physiologique, de haut niveau) à partir d'une carte de la radioactivité présente dans le système sanguin, notamment au niveau du cœur. La raison pour laquelle les médecins ont de bonnes raisons de croire, face à cette image de TEMP, qu'ils observent la perfusion myocardique est que la substance radioactive qui a été injectée, généralement du thallium 201, a la particularité d'être transportée par le sang jusqu'aux cellules du myocarde sur lesquelles elle se fixe. Or, si celui-ci n'est pas correctement irrigué, en raison de problèmes artériels, la concentration de thallium sur la paroi est moindre, dans le cas d'une hypoperfusion du myocarde, jusqu'à être inexistante dans le cas d'une nécrose sévère. C'est donc l'affinité du thallium 201 avec les fibres musculaires, connue empiriquement, qui est à la base de l'explication. D'autres contraintes doivent être prises en compte, telle la quantité de radiotraceur administrée qui doit être suffisamment petite pour ne pas perturber le phénomène en cours d'observation, ou le fait que la molécule utilisée puisse être facilement évacuée de l'organisme dans les heures 
qui suivent. Ce sont donc des arguments de nature essentiellement empirique qui sont employés ici à la justification qu'il existe une dépendance directe entre le rayonnement détecté dans le tissu musculaire et la perfusion de ce tissu.

Pour notre deuxième exemple, nous nous tournons vers le cas longuement décrit et analysé par le philosophe Dudley Shapere (1982) concernant l'observation du noyau solaire par la détection de neutrinos produits par celui-ci. De longs développements sont destinés dans cet article à établir qu'une bonne carte de l'émission de neutrinos est obtenue par un détecteur approprié, mais nous nous intéressons particulièrement ici à la propriété de haut niveau que les astrophysiciens prétendent observer, à savoir la production d'énergie dans le noyau solaire, à partir de cette carte d'une propriété de bas niveau: l'émission des neutrinos dans ce même noyau solaire. D'après lui, «les physiciens et astronomes ont développé une théorie qui, sur la base de nombreuses et diverses considérations, semble très bien rendre compte de la production d'énergie dans les étoiles» (ibid.: 493; notre traduction) ${ }^{8}$. Puisque cette théorie relie le taux d'émission de neutrinos (propriété de bas niveau) à la production d'énergie (propriété de haut niveau) à travers une loi quantitative, les physiciens et astronomes peuvent déduire de manière fiable la quantité d'énergie produite dans le noyau solaire à partir du nombre de neutrinos ayant été détectés. Il faut noter aussi l'indépendance entre ce que Shapere appelle la «théorie de la source» (cette loi quantitative), qui est la meilleure connaissance disponible concernant le mode de production des neutrinos dans le noyau solaire - et tisse le lien entre propriétés de bas et de haut niveau -, et les théories de la transmission et de la détection, qui décrivent le chemin des neutrinos dans l'espace et leur détection par un instrument, sans se préoccuper des conditions de leur production. Nous retenons que, dans cet exemple, contrairement au précédent, la dépendance des propriétés de bas et de haut niveau est justifiée de manière théorique.

Ces exemples démontrent que, pour rendre compte de manière générale des situations dans lesquelles les scientifiques affirment observer des phénomènes, il serait sans doute judicieux d'examiner deux aspects du problème. Le premier est le rôle que joue l'explication dans l'observation, rôle que les philosophes ont eu tendance à évincer pour mettre en avant l'immédiateté de l'expérience dans l'observation. On conçoit bien qu'un concept d'observation, dans lequel l'explication est nécessaire, induit une confusion dans la distinction traditionnelle entre théorie et observation. Pourtant, si nous voyons déjà dans la complexité croissante des systèmes d'imagerie de sérieuses raisons pour devoir fournir une explication relative à la transmission et à la détection d'une information dans le cas où un instrument est utilisé, une explication apparaît indispensable, que l'on utilise ou non un instrument, dès lors que c'est une propriété de haut niveau que l'on cherche à observer. Dans ce cas, en effet, il faut justifier la dépendance entre cette propriété et les propriétés de bas niveau, c'est-à-dire celles que l'on observe directement à partir des données acquises. Par suite, si l'on accepte que l'explication joue un rôle dans l'observation, un deuxième aspect du problème est de rendre compte de la diversité des propriétés que les scientifiques tentent d'observer. Dans une tâche d'une telle ampleur - puisque ces propriétés sont, on l'a vu, propres à chaque domaine scientifique -, une taxonomie de l'explication scientifique sera sans doute bien utile pour décider quand une justification est acceptable concernant la dépendance de propriétés de bas et de haut niveau. Nous n'allons cependant pas plus loin sur ces questions, que nous ne soulevons ici qu'à titre programmatique, et tirons maintenant les conclusions de ce travail.

Nous avons tenté de mieux rendre compte du contenu des images scientifiques produites avec des instruments, en nous appuyant sur le concept d'observation. Ce choix était motivé, nous l'avons dit, par l'utilisation qui est faite de ces images comme preuves concernant des phénomènes et par l'emploi fréquent du terme "observation" par les scientifiques qui produisent ces images. Notre critique des positions philosophiques sur le sujet n'a finalement constitué qu'un détour pour revenir à notre point de départ, c'est-à-dire la définition de l'observation 
de Claude Bernard («la constatation exacte d'un fait à l'aide de moyens d'investigation et d'études appropriées à cette constatation»), en précisant à quoi les faits dont il parle peuvent se rapporter. La recommandation de Claude Bernard, «de prendre toujours pour point de départ d'une expérimentation ou d'un raisonnement un fait précis ou une bonne observation, et non un mot vague» (1966: 285), méritait à notre sens d'être appliquée à l'observation elle-même et, à travers ce concept, à la lecture des images scientifiques produites par des instruments. Lire une image, concluons-nous, c'est non pas identifier une entité par la reconnaissance de quelque forme particulière, mais plutôt se donner un contexte d'investigation mettant en jeu une propriété ou un phénomène précis, et décider si l'image apporte des éléments dans la connaissance de ces phénomènes.

\section{NOTES}

1. Il s'agit là des cas d'observation à travers une loupe, des jumelles, des lunettes, etc., qui sont en quelque sorte des instruments « de confort", permettant de voir avec moins d'effort ce que nous verrions autrement en nous penchant plus près d'un document pour le déchiffrer ou en nous rapprochant d'un objet lointain.

2. L'article de Ian Hacking, auquel nous nous référons ici, s'intitule "Do we see through a microscope?» et a été traduit en français sous le titre «Est-ce qu'on voit à travers un microscope?». Dans le registre littéraire, on peut également mentionner La Montagne magique de Thomas Mann, œuvre dans laquelle il est fait référence aux radiographies comme étant des "photographies intérieures".

3. Ces propriétés en nombre très limité sont réminiscentes à l'échelle macroscopique de celles autorisées par la «nouvelle science» au XVII ${ }^{e}$ siècle, pour combattre une certaine dérive tautologique héritée des traditions scolastique et aristotélicienne. Plutôt que d'expliquer l'effet apaisant de l'opium par sa «vertu dormitive», les scientifiques de l'époque préféraient trouver une explication faisant intervenir les seules propriétés de taille, forme, position et mouvement des corpuscules élémentaires de matière. Ainsi l'effet apaisant de l'opium pourra-t-il s'expliquer par la forme ronde de ses particules, qui tranquillise le nerf en roulant sur lui (voir, par exemple, T. Kuhn [1970: 148-149]). 4. "What are the entities on either side of the divide between the observable and the unobservable?».

5. «Disputes about what is real frequently turn on objects or kinds of objects: Do prions or cartels really exist? Is there a planet beyond Neptune? What evidence was there in the late nineteenth century for the existence of atoms? Do emotions exist separately from their associated brain states? And so on. Stock examples can be misleading, for issues in realism also concern existence of properties: how do we know that quantum spin exists? Is there such a property as alienation in society? Is covalent bonding a specifically chemical property? [...] The entire debate on observables has been biased by an excessive focus on size and relative distance as surrogate measures for the degree of difficulty of observing objects".

6. "The discovery process for «objects » consists in specialized kinds of instruments detecting one or more of the properties that constitute what we consider to be that object with, subsequently, more and more properties constituting that entity being detected through further instrumental techniques".

7. À partir de ces propriétés physiques détectées, nous avons une connaissance directe des propriétés spatiales de l'objet étudié, en identifiant des formes dessinées par les variations de ces propriétés physiques sur l'image. Nous comptons donc également les propriétés spatiales comme étant des propriétés de bas niveau.

8. «Physicists and astronomers have developed a theory which, on the basis of a great many diverse considerations, appears to give an excellent account of the production of energy by stars" .

\section{RÉFÉRENCES BIBLIOGRAPHIQUES}

BERNARD, C. [(1865) 1966]: Introduction à l'étude de la médecine expérimentale, Paris, Garnier-Flammarion.

FrAASSEN, B. C. van [1980]: The Scientific image, New York, Oxford University Press.

HACKING, I. [(1981) 2004]: Philosophie des sciences, naturalismes et réalismes, tome II, Paris, Vrin, 238-274.

HumphreYs, P. [2004]: Extending Ourselves, Computational Science, Empiricism and Scientific Method, New York, Oxford University Press. KUHN, T.S. [(1970) 1983]: La Structure des révolutions scientifiques, trad. de L. Meyer, Paris, Flammarion.

SHAPERE, D. [1982]: "The concept of observation in science and philosophy", Philosophy of Science, n ${ }^{\circ} 49,485-525$. 